\title{
ORRMS DOPPELKONSONANTEN.
}

In dieser alten streitfrage herrschen noch verschiedene meinungen. Die von Sweet, Morsbach u. a. verfochtene ansicht, dafs die doppelkonsonanten die kürze des vorhergehenden vokals anzeigen sollen, ist zwar wohl in diesem augenblick die unter den fachgenossen vorherrschende, aber noch im jahre 1904 wurde die ansicht Trautmanns, wonach Orrm so schrieb, wie er im verse sprach, und also, was doppelt geschrieben erscheint, auch lang gesprochen wurde, mit grofser energie in einer Marburger dissertation von Peter Lambertz verteidigt und weiter begründet.

Es hat in dieser frage nur ein entweder - oder gegeben: entweder bezeichnen die doppelkonsonanten die kürze des vorhergehenden vokals oder die länge des konsonanten selbst, und es dürfte wohl aussehen, als ob es ein drittes nicht gäbe. Meiner meinung nach hat es aber ein drittes gegeben: es fragt sich nämlich einerseits was Orrms ursprünglicher plan war und andererseits, wie dieser plan zur ausführung gelangte. Wenn man diese beiden gesichtspunkte genügend berücksichtigt, wird man meiner meinung nach finden, dals weder Sweet, Morsbach usw. noch Trautmann, Lambertz usw. völlig recht gehabt haben. Es wird sich auch herausstellen, dafs keiner von ihnen völlig unrecht gehabt hat.

Für die mittelenglische grammatik dürfte der streit als ein streit um des kaisers bart aussehen. Für uns ist ja vor allen dingen die tatsache von gewicht, dafs wir dank den doppelkonsonanten Orrms über seine vokalquantitäten im grofsen und ganzen sehr genau unterrichtet sind. Was Orrm mit seinen schreibungen beabsichtigte, ist und bleibt ja nebensache. 
So ganz ohne interesse ist die frage aber nicht. Wie aus der darüber geführten diskussion hervorgehen dürfte, berührt sie sich nämlich mit mehreren anderen fragen, die für unsere auffassung der lautlichen verhältnisse unseres denkmals von gewicht sind oder aber von gewicht sein können. Es wäre auch erfreulich, wenn der streit einmal aus der welt geräumt werden könnte.

Ein referat über die diskussion dürfte hier am platze sein. Das hat aufserdem den vorteil, dafs ich dadurch in den stand gesetzt werde, ohne all $\mathrm{zu}$ weitläufige argumentation meine eigene meinung vorzutragen.

Auf die ansichten älterer gelehrter wie Wanley, Tyrwhitt, Conybeare, Turner, Thorpe brauche ich hier nicht einzugehen. ${ }^{1}$ )

Ellis, On Early Engl. Pron. (1869) s. 55 und 486, ist der ansicht, dafs Orrms doppelkonsonanten lediglich den zweck haben, die kürze des vorhergehenden vokals zu bezeichnen.

Ähnlich spricht sich auch Jessen, Tidsskrift f. phil. og pæd. I (1860) s. 203 aus.

Sweet, Hist. of Engl. Sounds (1874) s. 48, ist derselben meinung, findet es aber unerklärlich, dals Orrm z. b. witenn 'wissen', sune 'sohn' schrieb, obgleich in diesen wörtern zweifellos der vokal kurz war.

ten Brink, Zeitschr. f. d. Altertum XIX s. 213 (1876) sagt u. a.:

"Es ist bekannt, dais Orrm vokalkürze durch gemination des folgenden konsonanten ausdriickt; jedoch hat man, wie es scheint, nicht gesehen, in welchen grenzen er sich dabei hält. Orrm rerdoppelt nämlich nur den oder den ersten der konsonanten, welche eine silbe auslauten: enngell, Godd, nicht dagegen den einfachen konsonanten zwischen zwei vokalen. So schreibt er flocc, flocces, dagegen Godd, Godess ....... Wenn demnach Sweet es als 'a formidable difficulty' bezeichnet, dafs Orrm niemals wittenn, sunne (ae. sunu) schreibt, so sehen wir, wie diese schwierigkeit in nichts zerrinnt."

Trautmann, Anglia Anzeiger VII s. 94-99 (1884) bekämpft die von den genannten gelehrten ausgesprochene ansicht. Er geht von der nunmehr schon längst als richtig anerkannten tatsache aus, dals in Orrms sprache die me. vokaldehnung in offener silbe noch nicht eingetreten war

1) Siehe darüber Effer, Anglia Beiblatt VII s. 166. 
"Da nun Orrm rund 150 wörter vom schlage name, witenn, berenn hat, die er zusammen mindestens 3000 mal gebraucht, und da er in keinem einzigen dieser fälle nach unzweifelhaft kurzem vokal verdoppelten konsonanten setzt, so kann die regel "kürze des vorhergehenden vokals wird durch doppelten konsonanten ausgedrückt' nicht für ihn bestanden haben."

Da nun Orrm in übereinstimmung mit dem Altenglischen wörter wie faderr, sune, witenn, oferr mit ein em konsonanten, dagegen wörter wie sunne, sinne, spellenn, alle, sette, messe mit doppelkonsonanten schreibt, so schliefst Trautmann: "Orrm unterschied, gerade wie das Altenglische, zwischen kurzen und langen konsonanten und bezeichnete die kurzen durch einfache und die langen durch doppelte buchstaben."

Die fälle, in welchen Orrm, abweichend vom Altenglischen, in hochtoniger silbe doppelkonsonanten schreibt, teilt Trautmann in vier kategorien ein:

1. wo im Altenglischen der konsonant tatsächlich lang war: mann, bedd, bridd, sibb u. dgl. m.

2. Wörter wie shall, loff, Godd, gripp, staff, in welchen der auslautende konsonant im Altenglischen kurz war.

3. Wörter wie purrh, hallf, crafft, wurrplike, in welchen der erste von zwei konsonanten, die ein wort oder eine silbe auslauten, doppelt geschrieben wird.

4. Wörter wie wrihhte, pannkess, sammnenn, bollzhenn, in welchen der auslaut der silbe doppelt geschrieben wird.

Aus diesen verhältnissen zieht nun Trautmann den folgenden schlufs: "Für Orrm galt ein silbenauslautgesetz, wonach jeder eine silbe schliefsende konsonant nach kurzem vokale lang (und nach langem vokale kurz) war."

Auch nach unbetonten vokalen nimmt Trautmann, wenn auch zögernd, langen konsonanten an in fällen wie filledenn, kaseress, affterr, Cristess; sicher sprach er in solchen fällen lange konsonanten "beim lesen seiner eben und würdig einherschreitenden verse".

Trautmann fafst seine ansicht in den folgenden worten zusammen: "Orrm schreibt nicht einfachen konsonanten 
um länge, und nicht doppelten um kürze des vorhergehenden vokals auszudrücken, sondern er schreibt auf grund des gesetzes: 'Konsonantischer silbenauslaut ist kurz nach langem und lang nach kurzem vokal'."

Ich brauche wohl das Trautmannsche quantitätsgesetz hier nicht weiter zu erörtern. Es glauben wohl heut zu tage nur wenige daran in der form, in welcher er es formuliert, abgesehen von fällen wie shall, loff, Godd, gripp, staff, in welchen der konsonant zweifellos lang war. Nur möchte ich bemerken, dals fälle wie burrh, hallf, crafft usw. von seinem gesetz nicht betroffen werden, denn in diesen steht ja der lang geschriebene konsonant nicht im silbenauslaut. Man gewinnt aber den eindruck, dafs Trautmann auch in diesen fällen den konsonanten für lang hielt. Dafs er aber solche fälle bei der formulierung seiner regel nicht mit einbegriff, deutet wohl darauf hin, dals er sich hier einigermaisen unsicher fühlte. Soviel scheint mir sicher: auch derjenige, welcher an sein quantitätsgesetz glaubt, mufs hier einen wunden punkt finden.

H. Effer, Anglia Anzeiger VII s. 166-199 (1884), bringt eine ausführliche und nützliche materialsammlung zur aufklärung des problems. Wie Trautmann, nimmt er daran anstols, dafs Orrm nach kurzem vokal in offener silbe einfachen konsonanten schrieb: "Nehmen wir an, Orrm habe vokalkürze durch doppelung des folgenden konsonanten ausdrücken wollen, so drängt sich doch die frage auf, weshalb Orrm von dem system seiner schreibung abweicht und bei altenglisch kurzen vokalen in offener silbe eine neue bezeichnung für die kürze das kürzungshäkchen einführte, da er doch hier die kürze ebenso gut durch verdoppelung des folgenden einfachen konsonanten hätte ausdrücken können, wie dies regelmälsig in den anderen fällen nach kurzen vokalen geschieht." Hält man an der ansicht fest, die doppelten konsonanten bezeichnen kürze des vorhergehenden vokals, so kann nach Effer von einem einheitlichen system, welches Orrm seiner schreibung zu grunde gelegt hat, nicht mehr die rede sein. Orrm bezeichnete mit seiner konsonantenschreibung die quantität der konsonanten.

In bezug auf die schreibungen nach unbetonten vokalen teilt Effer nicht ganz die ansicht Trautmanns: "Da nach 
vokalen in untreffigen silben dieselbe art der schreibung beobachtet ist wie in treffigen silben, so kann man wohl annehmen, Orrm hat in seinem streben, eine möglichst einheitliche schreibung überall durchzuführen, die konsonantenschreibung in treffigen silben in gleicher weise auf die untreffigen übertragen, so dafs hier auf die aussprache der konsonanten kein besonderes gewicht gelegt zu werden braucht."

Kurz vor Effers aufsatz erschien die abhandlung von Erik Brate, Nordische Lehnwörter im Orrmulum, PBB. X s. 1 ff.: auch hier wurde die Orrmsche schreibweise erörtert. Brate schliefst sich der älteren auffassung an: "bekanntlich wird in geschlossenen silben die länge des vokals durch einfache, die kürze durch doppelte setzung des konsonanten ausgedrückt."

Die doppelschreibung eines konsonanten bezeichnet weiter nach der ansicht Brates altenglische gemination oder langen konsonanten (z. b. irre); die verbindungen $z z$ und $w w$ bedeuten vielleicht $i i$, un. Zwischen zwei vokalen bezeichnet die doppelschreibung in einigen fällen (wrappen, immess) vielleicht die länge des ersten vokals.

Im nachtrag zu seinem obengenannten aufsatz (s. 199) kritisiert Effer die ansicht Brates. Sie ist seiner meinung nach ein beweis dafür, dafs das einheitliche system, welches Orrm in seiner schreibung durchgeführt hat, vollständig fallen mufs, so lange man die alte ansicht, dafs doppelschreibung des konsonanten die kürze des vorhergehenden vokals ausdrückt, bestehen läIst. Mit recht weist Fffer die ansicht Brates bezüglich wrappen, immess als unhaltbar rurück.

In seinem 1884 erschienenen buche, Chaucers Sprache und verskunst s. 65 (2. aufl. s. 64), gibt ten Brink Trautmann insofern recht, als er die doppelschreibung der konsonanten mit der konsonantendehnung im wortauslaut nach betontem kurzen vokal in verbindung setzt. Orrms system hält er aber für unvollkommen "insofern, als er unbetonte silben nicht anders behandelt denn betonte. Auch darin wird es schwerlich der sache entsprechen, dafs er, wenn im wortauslaut mehrfache konsonanz auf kurzen vokal folgt, den ersten der betreffenden konsonanten gleichfalls doppelt".

Von einer solchen unvollkommenheit will aber Trautmann nichts wissen. In der Anglia, Anz. VII s. 209 (1884) will er gegen 
ten Brink (und Effer) geltend machen, dafs Orrm auch in unbetonten silben (z. b. ridenn) lange konsonanten sprach. $\mathrm{Er}$ hält es auch für sicher, dafs Orrm überall, wo er von zwei auslautenden konsonanten den ersten doppelt, diesen auch lang aussprach. Fingerzeige dafür gibt ihm das Neuenglische.

Sweet, First Middle Primer (1884; zweite auflage 1895), bleibt bei seiner alten ansicht, dafs die doppelkonsonanten zum zweck haben "to show the shortness of the preceding vowel .... whenever the consonant is final or followed by another consonant".

Seine ansicht führt er in der zweiten auflage seiner History of English Sounds (1888) s. 165 weiter aus. Charakteristisch für seine auffassung sind die folgenden worte: "That Orrms doubling did not mean cons. length, but had been reduced to an abstract symbolization of vowel length as in Modern English and German, is clear from his extension of it to unstrest syllables .... Where the consonant is followed by a vowel, as in sune $=0 \mathrm{E}$. sunu, it was not possible to double the consonant because it would then have been pronounced double, and sune would have been confounded with sunne 'sun' .... Here, then, Orrms clumsy spelling breaks down completely, and he feels this himself, for he often marks the shortnes of the vowel in such words as sune with a $(\checkmark)$ as in tăkenn, năme, chĕle.

Sweets ansicht wird von Morsbach in seiner Mittelenglischen grammatik (1896) s. $32 \mathrm{ff}$. geteilt und weiter begrïndet. Die von Trautmann vertretene ansicht wird von Morsbach in gründlicher weise kritisiert. Seine auffassung geht aus den folgenden worten zur genüge hervor: "Wenn Orrm wirklich zunächst nur die konsonantenlänge bezeichnen wollte, so hätte er nicht die doppelzeichen auch in tausend anderen fällen gesetzt, in denen der konsonant wirklich kurz war. Dadurch hob er seine regel tatsächlich wieder auf." Und: "In geschlossener silbe konnte die doppelschreibung der konsonanten zu keinen irrtümern in der aussprache anlals geben. In betonter silbe waren ja alle auslautenden oder die silbe zunächst schliefsenden konsonanten tatsächlich lang. Wenn er diese orthographische regel, die sich lautgeschichtlich gleichsam von selbst ergeben hatte ....... auch auf die unbetonten silben übertrug, so war diese konsequenz nur dann 
gerechtfertigt, wenn er mit den doppelzeichen lediglich vokalkürze, nicht aber konsonantenlänge bezeichnen wollte."

Gegen Sweet und Morsbach wendet sich nun Trautmann, Anglia XVIII (1896) s. 371-381. Er behauptet, das Alt- und Mittelenglische habe, wie das Neuenglische, lange konsonanten auch in "untreffigen" silben gehabt, sobald diese gewicht erhielten. "Und waren dem Mittelenglischen lange konsonanten auch in untreffiger silbe geläufig, so sind wir genötigt anzunehmen, dafs Orrm mit seinen doppelzeichen, durch die er in treffigen silben stets konsonantenlänge ausdrückt, auch in untreffigen konsonantenlänge meine. Nun hat sicher die doppelung der auslautenden konsonanten nicht blofs gewisser sondern aller untreffigen silben auf den ersten blick etwas befremdliches; doch nur auf den ersten blick, und sie ist keineswegs unerklärlich: Orrm schreibt in versen und sprach und wollte jede silbe seines eben und würdevoll einherschreitenden verses mit nachdruck gesprochen wissen."

Diese hypothese will Trautmann nun mit allerlei, wie mir scheint, sehr zweifelhaften metrischen erwägungen weiter begründen. Unter anderem nimmt er an, dafs Orrms versmals in dem grade von dem lateinischen hymnenverse abhängig sei, dafs er auch die sonst unbetonten silben im verse starktonig las: "Verstärkte sich aber das gewicht der untreffigen silben, d. h. werden sie treffigen ähnlich und sogar gleich, so mufsten sie den ausspracheregeln der treffigen unterliegen und handelte Orrm blofs folgerichtig, wenn er nu broperr Wallterr broperr min schrieb."

Trautmann hebt weiter hervor, dals wörter wie wezzes, trowwen $250 \mathrm{mal}$, oder öfter, im versausgang stehen, also lange stammsilbe enthalten müssen; $\bar{\delta}, w w$ können also nicht blofs den zweck haben, die kürze des vorhergehenden vokals anzudeuten, sondern sind intervokalischem $m m, t t, s s, f f$ usw. gleichwertig. Er kommt dabei aber zu dem etwas verblüffenden resultate, dals $z \xi$ und $w w$ lange vokale $(\bar{\imath}$ bezw. $\bar{\imath})$ bezeichnen. ${ }^{1}$ )

1) Gegen Morsbach (s.33f.): "Dals Orrm nicht die länge oder kürze der konsonanten, sondern der vokale bezeichnen wollte, geht zur gewifsheit aus 
In den Engl. Stud. XXVI (1899) s. 455 f. wird unsere frage von Mc Knight kurz gestreift. Nach ihm beweisen formen wie offe, onne am versende, dafs "Orrm used double consonant to indicate long consonant even in closed syllable", was aber nichts neues ist, da es, auf grund solcher wörter wie manne, cunnenn, wille, spelle am versende, schon vorher allgemein als richtig anerkannt war. Wahrscheinlich hat aber Mc Knight damit sagen wollen, dals onne, offe beweisen, dals die konsonanten in onn, off lang sein müssen, und darin hat er vollkommen recht.

Ich gehe jetzt $z u$ der oben erwähnten dissertation von Peter Lambertz, Die Sprache des Orrmulums nach der lautlichen Seite untersucht (Marburg 1904) über, in welcher (s. 92-102) der verfasser die frage einer erneuten prüfung unterzieht. Er bespricht nur die fälle, wo anscheinend ohne sprachgeschichtliche rechtfertigung buchstaben doppelt geschrieben sind, nämlich 1 . in den diphthongen, 2. in wörtern wie wipputenn, 3. im auslaute unbetonter silben und wörtchen.

1. Die schreibung sizze liest Lambertz als sti-je, dazzes als dai-jes, wezze als wei-ie (dagegen wrēzenn als wré-jenn), tawwenn als tau-uenn, chewwenn als cheu-uenn usw. Im silbenauslaut wie vor konsonanten liest er mit Trautmann azz $(=a \bar{\imath}), e i(=e \bar{\imath}), a w(=a \bar{u})$ usw. Er schliefst daraus (s. 100), "daIs keinem zeichen ein blols diakritischer wert zukommt, dals also jeder buchstabe auch seinen lautwert besitzt. Demnach gilt hier voll und ganz die theorie Trautmanns. A priori liefse sich sein system ebensowohl auf die doppelkonsonanten anwenden. Aber es hält auch einer genaueren prüfung stand, und alle einwürfe, die dagegen gemacht worden sind, hat Trautmann widerlegt".

2. Wörter wie wipputenn, efennald, onnan liest L. mit einem knacklaut am anfang des letzten kompositionsgliedes.

3. Bei Orrm müssen sehr oft satztieftonige wörter betont gelesen werden; es kommt auch nicht selten vor, dafs vor- und nachtonige silben zur tonsilbe erhoben werden.

seiner schreibung der diphthonge hervor: aw für āu neben awvo für au, aew für $a u$, ew, eow für eu neben ewow, eoww für eu, ow für $\delta u$ neben ovwo für ou, ferner $a z z$ für $a i$, ezz für ei z. b. in sāwle, clawwess, shawenn, newe, cnewowe, flowenn, trowwenn, dazz, wezze. 
Dieser umstand soll nach Lambertz die annahme Trautmanns noch mehr stützen, dafs Orrm "jede silbe .... mit nachdruck gesprochen wissen wollte". Er ist davon überzeugt "dafs Orrm schrieb, wie er im verse sprach".

Ich gehe jetzt zur vorlegung meiner eigenen ansicht über. Meiner meinung nach ist die sache viel einfacher als vorher angenommen worden ist, und eine durcharbeitung der einschlägigen, von mir kurz referierten literatur, hat mich in dieser auffassung bestärkt.

Die frage nach der aufgabe und bedeutung der Orrmschen doppelkonsonanten ist nicht nur eine sprachliche, sondern auch eine psychologische. Es handelt sich nämlich nicht nur darum, wie Orrm sprach, sondern selbstverständlich vor allen dingen darum, wie er $\mathrm{zu}$ sprechen glaubte.

Wenn wir feststellen können, wie Orrm zu sprechen glaubte und dafs seine orthographie damit übereinstimmt, dann dürfte der wahrscheinlichkeitsbeweis dafür erbracht sein, dafs seine absicht war, eine rein phonetische schrift zu schaffen, dals also seine doppelkonsonanten zur bezeichnung langer konsonanz dienen sollten, und dafs sie nicht zur aufgabe hatten, die kürze des vorhergehenden vokals zu bezeichnen.

Wie glaubte nun Orrm zu sprechen? Lälst das sich wirklich feststellen? Wie eigentümlich es aussehen mag, ich glaube, diese frage ist nicht unlösbar. Wir müssen nur an personen, die in ihrer sprache ungefähr dieselben quantitativen verhältnisse haben, wie seinerzeit Orrm, und die ungefähr dieselbe sprachliche bildung genossen liaben, experimente machen. Vor allen dingen dürfen unsere versuchspersonen die phonetischen abhandlungen von Sweet, Trautmann, Viëtor usw. nicht gelesen haben; das hatte ja auch nicht Orrm. Um die frage zu lösen, muls man meiner meinung nach auf sie etwas mehr bauernverstand und etwas weniger gelehrsamkeit anwenden.

Das für unsere frage wichtigste und zugleich das leichteste experiment will ich hier gleich erörtern. Wie seiner zeit Orrms sprache, so hat auch das moderne Englisch, Deutsch, Schwedisch usf. in wörtern von dem typus deutsch haben, flegel, wasser, engl. ended, business, basket, schwed. vagnen, 
skeppet, Moses, Magog kurze konsonanten im unbetonten auslaut; diese wörter stehen also, soweit in ihnen der vokal der endsilbe ausgesprochen wird, mit Orrms nemmnedd, limess usf. ganz auf einer linie.

Fragen wir nun einen Engländer, Deutschen oder Schweden, der zwar eine gute bildung genossen hat, aber von der modernen phonetik nichts weils, ob der endkonsonant in diesen wörtern lang oder kurz sei, so antwortet er meiner erfahrung nach in der regel: lang. In den wenigen fällen, in welchen die antwort anders lautet, müssen wir damit rechnen, dafs unser gewährsmann sich von dem schriftbild hat beeinflussen lassen.

Wie kommt es nun, dais phonetisch ungeschulten die tatsächlich kurzen endkonsonanten unbetonter schlufssilben lang vorkommen? Auf diese frage gibt es, glaube ich, nur eine antwort. Wenn ein phonetisch ungeschulter in die lage kommt, sich über die quantität der konsonanten in unbetonten silben äufsern zu müssen, dann mufs er die betreffende silbe näher untersuchen; dies geschieht dadurch, dafs er die silbe isoliert ausspricht; das ist für ihn der einzige ausweg, die silbe so zu sagen unter die lupe zu nehmen. Eine isoliert ausgesprochene silbe ist aber in der regel starktonig; in starktoniger stellung gibt es im wortschlufs in dem modernen Deutsch, Englisch oder Schwedisch nicht die verbindung von kurzem vokal + kurzem endkonsonanten. Dieselben verhältnisse herrschten in Orrms sprache. Es boten sich ihm nun zwei alternativen: die isolierte silbe entweder als kurzen vokal + langem konsonanten, oder als langen vokal + kurzem konsonanten auszusprechen, denn nur diese lautverbindungen waren seiner sprache in starktoniger stellung im wortauslaut geläufig. $\mathrm{Er}$ mulste also -nĕd in nemned entweder als néd oder als nedd aussprechen. Von diesen beiden auswegen kam ihm der letztere als der bessere vor, wahrscheinlich weil die vokalquantität sich leichter feststellen liefs als die konsonantenquantität. Dasselbe gilt auch für den modernen Engländer, Deutschen oder Schweden. Ausnahmsweise können wir aber in solchen silben einen langen vokal beobachten bei ungebildeten, die "fein" sprechen wollen, oder bei ausländern, z. b. einem Engländer, der deutsch oder schwedisch lernen will. Ich habe 
öfter bei Engländern die aussprache hābēn (neben hābĕnn) beobachtet. 1)

Schon a priori spricht gegen die annahme Sweets u. a., dals bei Orrm die doppelkonsonanz lediglich vokalkürze bezeichnete, der umstand, dals Orrm in diesem falle gegen seine regel in rund jeder siebenten kurzzeile hätte verstofsen müssen. Wenn Trautmann, Anglia Anz. VII s. 95 recht hat, hat ja Orrm mindestens 3000 schreibungen (in etwa 10000 septenaren) von dem typus name, witenn, berenn (vgl. auch z. b. filledenn, tācnede Trautmann s. 98), in welchen der vorausgehende vokal kurz war und in welchen er doch einfache konsonanten schrieb und schreiben mufste. ${ }^{2}$ ) Mit Trautmann muls ich bezweifeln, dals Orrm hier nach einander widersprechenden grundsätzen schrieb. Sogar Sweet findet seine eigene theorie wegen dieser tatsache gewissermafsen bedenklich - jedenfalls sieht er in ihr eine "formidable difficulty".

Nehmen wir also an, dafs Orrm mit seinen doppelkonsonanten wirklich lange konsonanten bezeichnen wollte. Wir wollen dann unter dieser voraussetzung versuchen uns klar

1) Ahnlich erklärt sich schwed. sinom (isoliert als sīnom ausgesprochen) in der phrase tusen sinom tusen 'tausend und aber tausend'. Im Altschwed. hiefs das wort sinnom (dat. pl. zu sinne oder sinn 'mal', das sonst im Neuschwedischen ausgestorben ist). In schwachtoniger stellung wurde daraus šnom (mit kürzung des konsonanten), und so heifst es noch in der natürlichen rede. Wer nun das wort isoliert oder haupttonig bei langsamem tempo aussprechen will, der kommt in ein dilemma, denn das Neuschwedische kennt unter dem hauptton nicht - wie das Deutsche oder Englische die verbindung von kurzem vokal + kurzem konsonanten + vokal. Es stehen also demjenigen, der das unbetonte sinom mit hauptton sprechen will, nur zwei wege offeu: entweder als sinnom oder sinom (sowohl sinnom als sinom würde in unbetonter stellung $\mathrm{zu}$ šnom werden). Von diesem hat man - vielleicht in anschlurs an das neuschwed. schriftbild - das letztere gewählt. Den ersteren ausweg wählen oft ungebildete, die schwed. telefón als tèllefón aussprechen und schreiben. - In ähnlicher weise wie sinnom ist schwed. $h \bar{\imath} n$ 'der teufel' (aus ȟn hále, hðn ónde 'der böse') entstanden.

2) Die ausnahmen sind änfserst selten: buttan (6336), abutten (9230; Effer, Anglia Anz. VII 8. 187), crummess (Effer 8. 185). Hierher gehören nicht wrabpenn 'to anger, offend', das sicher lange spirans hatte (siehe die beispiele bei Stratmann-Bradley; Einflufs des Sbst. wrappe, Erdmann bei Brate PBB. X s. 584, Björkman, Dialekt. Provenienz s. 12), immess (Björkman a. a. 0.), goddess, fattess, lottess (Effer s. 199).

Anglia, N. F. XXV. 
zu machen, wie wohl Orrm zu seinem system gekommen ist. Mir scheint der folgende arbeitsgang wahrscheinlich:

1. Zunächst mufs es dem methodischen Orrm inkonsequent vorgekommen sein, dals der lange konsonant im gen. mannes mit doppeltem konsonantenzeichen, im nom. mann aber mit einfachem konsonantenzeichen bezeichnet wurde. Eine geregelte englische orthographie gab es zwar zu Orrms zeit nicht, aber nach den erhaltenen engl. denkmälern aus dem 11. und 12. jahrh. zu urteilen, schrieb man wie im Altenglischen nur selten doppelkonsonanten im auslaut.

2. Da nun schon lange vor Orrms zeit alle auslautenden konsonanten nach betontem kurzem vokal gelängt waren, so versteht es sich von selbst, dafs er auch in staff sb., shapp sb., gladd adj., gripb sb., godd sb., sahh prät., usw. doppelten konsonanten schrieb. - Sie stehen ja alle mit mann, bucc, flocc in bezug auf die quantität des endkonsonanten auf einer linie, Dagegen schrieb er natürlich stafess pl., glade pl., godess gen., gripess gen., da $f, d, p$ usw. in diesen wörtern inlautend kurz waren. Sicher ist also, dals wir in wörtern von den typen mann, mannes, staff, stafess ohne schwierigkeit annehmen können, dals die quantität der konsonanten für die schreibung malsgebend war. Die schreibung stafess bedeutet also dann keine "formidable difficulty". ")

Dals Orrm demnach auch mannkinn, mannshipe, goddspell, gresshoppe schreiben mulste, ist ohne weiteres verständlich.

3. Der nächste schritt im entwicklungsgange des systems galt vielleicht anderen postvokalischen silbenauslautenden konsonanten als den eben behandelten. Es kamen hier zwei kategorien in betracht:

a) Fälle wie gill/te, wrohh/te, forr/me, dohh/ter, Orr/mulum, d. h. der konsonant steht postvokalisch im auslaut einer betonten, nicht wortauslautenden silbe.

b) Fälle wie kepenn, waterr, gledess, glaterrnesse, Cristess, selfennde, das präfix onn-, d. h. der konsonant steht postvokalisch im auslaut einer unbetonten silbe. $\mathrm{Zu}$ dieser kategorie können wir auch unbetonte wörtchen wie onn, off, patt, inn, iss, hitt, hiss zählen.

1) Ausnahmen wie goddess, lottess (statt godess, lotess) beruhen selbstverständlich auf formangleichung. Siehe Effer s. 184 f., 199. 
Was nun die fälle a) betrifft, so scheint es mir sicher, wenigstens wahrscheinlich, dafs die silbenauslautenden konsonanten hier wirklich lang waren; unter allen umständen waren sie länger, als nach langem vokal in dieser stellung (z. b. Cristess). Wenn sie lang waren, dann ist es ja ganz natürlich, dafs der systemgetreue Orrm sie auch lang schrieb. ${ }^{1)}$

Ich halte es für sicher, dafs Orrm in den fällen a) die konsonanten für lang hielt. Wenn wir aber beweisen können, dals sie tatsächlich lang waren, jedenfalls länger als nach langem vokal, dann wird es ja um so natürlicher, dafs Orrm sie als lang empfand und schrieb; dann wird auch die ehre Orrms als phonetiker - woran offenbar Trautmann so viel liegt - gerettet.

Zuerst müssen wir damit rechnen, dafs - wie die experimentalphonetischen forschungen der letzten jahre, namentlich die wertvolle arbeit von E. A. Meyer (Englische Lautdauer, Uppsala und Leipzig 1903), ${ }^{2}$ ) dargetan haben - weder alle langen noch alle kurzen laute die gleiche quantität (sei es länge oder kürze) haben, dals wir also keineswegs mit der üblichen dreiteilung der lautquantitäten in lang, halblang und kurz, geschweige denn mit der zweiteilung in lang und kurz, für alle wissenschaftlichen zwecke auskommen. Wie oben gesagt: es ist auf jeden fall anzunehmen, dafs die hier zunächst in frage kommenden konsonantenquantitäten länger waren, als nach den langen vokalen. An einem dürfen wir aber nicht zweifeln: Orrm hat die betreffenden konsonanten für lang gehalten und sie dementsprechend doppelt bezeichnet. ${ }^{3}$ )

Dafs die konsonanten in den fällen a) länger waren als nach langem vokal, beweisen die modernen germanischen sprachen, deren verhältnisse in dieser beziehung nicht auf

1) Vgl. Morsbach s. 34: "In geschlossener tonsilbe konnte die doppelschreibung der konsonanten zu keinen irrtümern in der aussprache anlafs geben. In betonter silbe waren ja alle auslautenden oder die silbe zunächst schliefsenden konsonanten tatsächlich lang."

2) Vgl. auch seinen aufsatz Vokaldehnung und Vokalqualität in Mod. Lang. Quarterly VI s. $140 \mathrm{f}$.

s) Es ist sehr möglich, dafs die menschen zu Orrms zeit ein feineres ohr für lautdauer hatten als wir: es genüge darauf hinzuweisen, dafs die modernen sprachen den unterschied zwischen $z$. b. sŭne und sŭnne nicht länger kennen. 
späterer entwicklung beruhen können, sondern gewiss den stempel der ursprünglichkeit an sich tragen.

Um gleich mit dem Englischen anzufangen, sei daran erinnert, dafs nach Meyer sowohl auslautende als inlautende konsonanten nach kurzem vokal länger sind als nach langem vokal, obgleich die inlautenden beträchtlich kürzer sind als die auslautenden. ${ }^{1}$ )

Im Deutschen herrschen ähnliche verhältnisse, wie mir dr. Meyer freundlichst mitteilt.

Schwedisches sprachgefühl faIst die konsonanten in dieser stellung als lang auf: z. b. strumpa, knektar, fyllde, doktor (die ungebildeten schreiben gewöhnlich docktor). In der tat ist aber in solchen fällen - gerade wie im Englischen - der konsonant etwas kürzer als im wortauslaut, zunächst also halblang (freundliche mitteilung Noreens). ${ }^{2}$ )

Ich vermute, dafs auch in den anderen modernen germanischen sprachen ähnliche verhältnisse herrschen, nehme aber an, dafs die drei sprachen (Englisch, Deutsch, Schwedisch)

1) Vgl. Jespersen, Lehrbuch der Phonetik 8. 181, wo nach Meyers ergebnissen die folgende tabelle beigegeben wird:

\begin{tabular}{c|c|c|c|c|c}
\hline & & \multicolumn{2}{|c|}{ A u s l a u t } & \multicolumn{2}{c}{ I n l a u t } \\
& Anlaut & $\begin{array}{c}\text { nach langem } \\
\text { vokal }\end{array}$ & $\begin{array}{c}\text { nach kurzem } \\
\text { vokal }\end{array}$ & $\begin{array}{c}\text { nach langem } \\
\text { vokal }\end{array}$ & $\begin{array}{c}\text { nach kurzem } \\
\text { vokal }\end{array}$ \\
\hline p & 11,5 & 12,6 & 14,8 & 8,0 & 10,2 \\
t & 11,2 & 10,1 & 11,9 & 7,9 & 9,0 \\
k & 10,5 & 12,0 & 13,3 & 8,9 & 10,6 \\
b & 10,0 & 8,8 & 10,1 & 6,2 & 7,1 \\
d & 9,1 & 6,2 & 7,9 & 4,9 & 5,7 \\
f & 11,2 & 13,1 & 13,5 & 7,3 & 8,7 \\
s & 13,2 & 14,1 & 14,5 & 9,3 & 9,5 \\
v & 10,3 & 9,8 & 10,5 & 4,9 & 4,8 \\
l & 10,6 & 13,6 & 17,4 & 7,2 & 7,5 \\
m & 10,2 & 15,5 & 17,8 & 7,9 & 8,6
\end{tabular}

2) In den älteren jahrgängen der "Svenska landsmålen" wurden diese konsonanten als lang bezeichnet. So schreibt z. b. Magnusson Sv. Landsm. II : burrk (s. 10), dyggd (s. 14), immpisska (s. 31). Nunmehr findet man diese bezeichnung im allgemeinen überflüssig. Vgl. aber noch Borgström, Askersmålets ljudlära 1913, wo die konsonanten in der betreffenden stellung als lang bezeichnet werden. 
genügen, um $\mathrm{zu}$ beweisen, dafs in der mittelalterlichen aussprache des Englischen wahrscheinlich so ziemlich dieselben quantitativen eigentümlichkeiten vorhanden waren.

Es ist auch anzunehmen, dafs die konsonanten Orrms in dieser stellung energischer als nach langem vokal ausgesprochen wurden. Dadurch wurde sicher der eindruck der länge noch deutlicher.

Die doppelkonsonanten in diesen fällen beruhen also so gut wie sicher darauf, dafs Orrm sie relativ lang aussprach, d. $h$. länger als in derselben stellung nach langem vokal. Wenn diese erklärung nicht an und für sich vollkommen ausreichte, so könnte man an eine andere denken: wenn Orrm eine silbe isoliert aussprach, mufste er den postvokalischen silbenauslautenden konsonanten notwendig lang aussprechen. Von dieser an und für sich möglichen erklärung können wir aber absehen, da wie gesagt, die andere vollkommen genügt.

Die fälle b) habe ich schon oben besprochen. Es lag dem guten Orrm wohl sehr daran, die quantität des schlufskonsonanten auszufinden, und das konnte er nun einmal nicht, ohne die endsilbe zu betonen.

Wie Morsbach s. 33 hervorhebt, werden auch im Neuenglischen auslautende konsonanten nach unbetontem vokal gelängt, "wenn die betr. endsilben aus irgend einem grunde (beim syllabieren, im satze bei gegenüberstellung etc.) mit stark emphatischem akzent gesprochen werden (in diesem falle werden die unbetonten silben überhaupt den betonten gleichgestellt)". Orrms methode um die quantität des end-n in kepenn festzustellen, war tatsächlich eine art syllabieren, wenn auch nicht nach Pestalozzis art '): bei seiner untersuchung der frage - aber nur dann - muls er sein kēpenn als képěnn ausgesprochen haben. Es fragt sich auch, ob nicht Orrm der begriff der silbe ganz geläufig war: bei seinen Lateinstudien kann ihm das wort syllaba kaum entgangen

1) Orrm und seine zeitgenossen bedienten sich wohl der uralten buchstabiermethode, bei welcher die silben isoliert ausgesprochen wurden. Leider findet sich hier in Uppsala, soviel ich weirs, keine literatur über die mittelalterlichen leselehrmethoden. Siehe dazu Meyers Konversationslexikon, artikel "Lesen". Auch Valentin Ickelsamers werke habe ich nicht einsehen können. 
sein, wie auch in altenglischer zeit der begriff allen Lateinkundigen bekannt war. 1) Daraus können wir schliefsen, daIs Orrm bei seinen experimenten nicht nur kē-pënn in einem worte, sondern auch pěnn isoliert ausgesprochen haben dürfte. ${ }^{2}$ ) Dafür spricht auch die schreibung fulizwiss (v. 11465, 7214), wo iz das bei isolierter aussprache der silbe entstandene lange $\bar{\imath}$ bezeichnet; es ist mit dem $\bar{\imath}$ in schwed. sinnom, hinn (oben s. 361 anm. 1) zu vergleichen.

4. Es erübrigt nur, die fälle zu besprechen, in welchen der konsonant nach kurzem vokal steht, ohne die silbe zu schliefsen, z. b. cnihht, crafft, twellf, purrh usw. Wie oben hervorgehoben, herrschen in solchen fällen in den modernen sprachen (wenigstens im Englischen, Deutschen und Schwedischen) genau dieselben quantitätsverhältnisse, wie wenn der konsonant im silbenauslaut steht. Wir haben keinen grund anzunehmen, dafs in diesen fällen zu Orrms zeit andere verhältnisse vorhanden waren. Wenn wir aufserdem bedenken, dafs Orrm auch in solchen fällen den konsonanten energischer als nach langem vokal ausgesprochen haben mufs, so kann es nicht wunder nehmen, dals er ihn für lang hielt.

Nehmen wir aber das durchaus unwahrscheinliche an, dals in diesen fällen der postvokalische konsonant kurz war. Dann hätte Orrm z. b. *cniht, plur. cnihhtess, *craft, plur. crafftess, eigentlich zu schreiben gehabt. Aber wir können gut verstehen, dals eine solche orthographie dem biederen Orrm unsystematisch hätte vorkommen müssen. Damit will ich selbstverständlich nicht gesagt haben, dals Orrm sich jemals mit einem solchen dilemma zu schleppen hatte. ${ }^{3}$ )

1) Vgl. Flfrics Grammatik und Glossar, hgg. von Zupitza (Berlin 1880) 8. 7: syllaba is stafżefêz on ûnre ordunge zeendod.

$\left.{ }^{2}\right)$ Ich gehe hier nicht auf die frage ein, ob die verwendung unbetonter silben unter dem ictus zu der Orrmschen orthographie beigetragen haben kann (Sammlungen bei Menthel, Anglia Beibl. VIII s. $81 \mathrm{f}$.). In vielen fällen dürfte taktumstellung anzunehmen sein. Andere fälle sind wohl dichterischem ungeschick (vgl. Schipper, Grdr. II 1. aufl. s. 1039) oder dem silbenzählenden charakter des Orrmulums zuzuschreiben.

3) Im satztieftonigen worte annd war das $n$ zwar kurz; für Orrm ist aber die lexikalische aussprache, wie bei onn, iss u. dgl. m., malsgebend gewesen. 
Damit sind die grundlinien der Orrmschen orthographie erledigt. Wie wir sehen, hat Orrm sein system mit grofser konsequenz durchgeführt. $\mathrm{Er}$ hatte mit keinerlei konflikten zu kämpfen - im gegenteil, alles klappt sogar in erfreulichster weise. Sweets "formidable difficulty" hat für Orrm nicht bestanden. Sein system ist in keinem punkte "zusammengebrochen", wie Sweet behauptet.

Wir müssen jetzt einige einzelheiten, die von den gelehrten in verschiedener weise gedeutet worden sind, näher ins auge fassen.

Zuerst drängt sich da die Orrmsche schreibung der diphthonge, aus welcher hervorgehen soll, dafs Orrm die länge oder kürze der vokale, nicht der konsonanten, bezeichnen wollte, 1) unserer aufmerksamkeit auf. Diese frage haben, glaube ich, Trautmann und Lambertz im grossen und ganzen richtig gelöst.

Wie Trautmann, Anglia XVIII hervorgehoben hat, kommen wörter wie wezzess, dazzess, trowuenn, cnewwess, $250 \mathrm{mal}$, oder öfter, im versausgang vor; die silbe muls also lang sein. Nur ist der von Trautmann aus dieser tatsache gezogene schlufs etwas ungeschickt formuliert: $z z$ soll nach ihm $\bar{\imath}$ und $w w \bar{u}$ bedeuten. Es hat sich zwischen dem stammvokal und dem mit $z$ bezw. $w$ bezeichneten laut (der zunächst als der halbvokal $i$ bezw. $u$ zu definieren ist) ${ }^{2}$ ) ein gleitlaut entwickelt. $0 \mathrm{~b}$ dieser gleitlaut mit $i, u$ (wie bei Lambertz) oder mit $i, \imath \mathrm{zu}$ bezeichnen ist, ob wir also weizness, trouuenn oder weizess, tronuen schreiben sollen, ist für unsere zwecke gleichgültig. Letzteres ist vielleicht jedoch vorzuziehen. ${ }^{3}$ )

Nur scheinbar ist die ausnahme -iz (z. b. in maniz): hier bezeichnet $-i z$ nämlich $\bar{\imath}$ (höchstens $\bar{\imath}$ ). Vgl. meine Scand. Loanwords s. 117 und die dort angeführte literatur; auch

1) Vgl. Morsbach, Me. Gr. s. 33.

2) Wie schwierig es ist, die grenzen zwischen einer spirans und einem halbvokal zu ziehen, beweist z. b. das moderne Schwedisch, wo $j$ bisweilen halbvokal, bisweilen eine spirans bezeichnet: nur ein sehr geschulter phonetiker kann entscheiden, in welchen fällen das eine oder das andere vorliegt.

3) Wenn ich $i$, un schreibe, will ich damit nicht gesagt haben, dafs die mit diesen zeichen bezeichneten lautgebilde durchaus homogen sind.

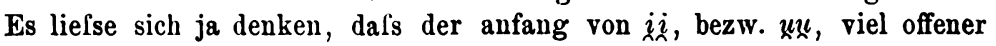
war als der schlufs. 
Lambertz, der meine ausführungen über die frage nicht erwähnt, setzt Orrms iz als $\bar{\imath}$ an. Dals demnach $i z z e$ als $\bar{i} i e$ aufzufassen ist, bedarf wohl keiner weiteren begründung. Vgl. Björkman a. a. 0.1), Lambertz a. a. 0.

Im auslaut und vor einem konsonanten ist ebenfalls $z \tilde{z}$,

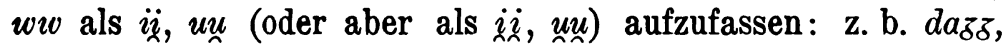
pezzre, lezztenn, sezzp, rezzn, wazzn, trowwpe, pewwtenn, nowwst, rowwst. Hier sind aber die ii, uu (ich möchte, wie gesagt, lieber $i i$, un schreiben) in anderer weise als zwischen vokalen entstanden: in $d a z z$ beruht $z z$ auf demselben konsonantendehnungsgesetze wie z. b. $d d$ in Godd, und in pezzre, $s e z z p$, trowwbe, nowwt sind $z z, w w$ in derselben weise wie $z . b$. hh in cnihht, cnihhtess zu beurteilen.

Dafs dagegen $\mathrm{zwischen}$ vokalen der anfang (oder die erste hälfte) des durch $z z$, ww bezeichneten phonems als ein gleitlaut aufzufassen ist, dafür spricht auch ein anderer umstand. Denn sonst wären schreibungen wie chewwenn, trowwenn, peowwess (daraus nom. peoww), prowwinng ${ }^{2}$ ) schwierig zu erklären: dagegen steht der annahme einer entwicklung von langem vokal $+w(\underline{u})$ zu langem vokal + gleitlaut $+w(u)$, woraus kurzer vokal + gleitlaut $+w(u)$ werden müfste, nichts im wege. Wenn aber nach langem vokal ein gleitlaut entstand, mufs unter sonst gleichen umständen auch nach kurzem vokal ein gleitlaut entstanden sein. Dagegen bin ich nicht davon überzeugt, dafs fälle wie trowwpe, mazzpe in derselben weise zu erklären sind, wie Lambertz s. 100 anninmt. Es ist ja doch sehr möglich, dals vor lautverbindungen wie $w p$, $z p$ vokalkürzung schon früher, und zwar zu einer zeit wo $z$ noch eine spirans war, hätte eintreten müssen, ${ }^{3}$ ) so dals diese kürzung an und für sich schon genügt, um die schreibung zu erklären.

Ich halte also Luicks annahme,4) dazzess sei als dai-es zu deuten, für weniger wahrscheinlich als daiįes (dain-ǐns).

1) Ich bezeichnete a. a. 0. den gleitlaut mit $z$; damit wollte ich mich nicht über die frage äufsern, ob dieser gleitlaut als halbvokal oder spirans aufzufassen ist.

$\left.{ }^{2}\right)$ Nach ae. $\bar{a}, \not$ wird intervokalisches $w$ nie verdoppelt, da cnawwesst Wh.-Holt. 12800, paewwess Wh.-Holt. 16911 usw. zu streichen sind. Vgl. Effer, Anglia Anz. VII s. 188.

s) Hat sāzole sein $\bar{a}$ aus der unflektierten form bezogen? Oder ist diese form mit der tatsache in zusammenhang zu bringen, dass $z$. b. kein *cnawwenn (sondern nur cnawenn) belegt ist?

4) Unters. s. 93. 
Das einfache $z$ in forrlezenn, forrlezerrnesse muis mit Luick a. a. 0., als auf einer störung der lautgesetzlichen entwicklung oder einem versehen beruhend erklärt werden. slazenn zu slān kann (wie forrlezenn) auf systemzwang beruhen (nach partizipien wie tredenn farenn).

Einen sonderfall bilden die wörter nowwhar, owwhar, owwhar. Die erklärung von Lambertz, wonach $w w$ in zwei teile zerfalle: "in dem zum vorhergehenden vokal gehörenden vokalischen und den die folgende silbe anlautenden konsonantischen", befriedigt mich nicht. Es ist ja so gut wie sicher, dafs schon zu Orrms zeit $h w$ zu einem stimmlosen $w$ geworden war, das Orrm mit wh bezeichnete. Langes stimmloses $w$ mulste Orrm mit wwh bezeichnen, gerade so wie er langes $\check{s}$ mit $s s h$ bezeichnete. Meines erachtens wollte Orrm mit $w w h$ hier langes stimmloses $w$ bezeichnen. Wie diese quantität nun zu erklären ist, darüber möchte ich mich nur mit dem gröfsten vorbehalt äufsern. Das $o$ war vielleicht schon altenglisch gekürzt: es stand ja dann vor einer konsonantengruppe. Es ist ja auch möglich, dals Orrm den aus $h w$ entstandenen laut, wenn er kurz war, in dieser stellung doch als lang auffalste, wozu seine zusammengesetzte natur hätte beitragen können. Vielleicht, ja wahrscheinlich, war der laut aber hier tatsächlich lang. Es liefse sich annehmen, dafs aus intervokalischem $h w$, wenn es zu stimmlosem $w$ assimiliert wurde, ein laut entstehen mufste, der dieselbe lautdauer in anspruch nahm, wie vorher das $h$ und das $w$ zusammengenommen. $\left.{ }^{1}\right)$ Man könnte auch vermuten, dals die konsonantenlänge auf der stellung vor nebenton beruht. Dafür würde gewissermafsen nuzzu sprechen, wo $z z$, wenn es nicht aus einem gleitlaut $+i$ (oder $z$ ) entstanden ist, kaum anders zu erklären ist.

Schreibungen wie wipputenn, wippinnenn, efennald, middelloerd, onnan, unnorne beruhen auf assoziation mit wipp, efenn, middell, onn, unn- usw. Es ist sogar möglich, wie Lambertz s. 101 hervorhebt, dafs hier utenn, innenn, ald usw. mit knacklaut anlauteten.

Wenn neben full iwiss öfter fuliwiss, fulizwiss steht, so

1) Das ww in owwperr, nowwperr ist dagegen sicher in derselben weise als z. b. das $p p$ in keppte zu erklären. 
müssen die schreibungen mit einfachem $l$ darauf beruhen, dafs der hauptakzent auf wiss lag und ful relativ oder ganz unbetont war, was natürlich kürzung des $l$ herbeiführen mufste. Nur durch anlehnung an full konnte eine aussprache mit langem $l$ entstehen. Die schreibung fulizwiss beweist uns obendrein, dafs Orrm mit der etymologie des wortes (denn er hat füliwiss sicher als ein wort ausgesprochen) nicht völlig vertraut war. ${ }^{1}$ )

Die schreibung efenninng (neben effeninng) beruht wohl auf anlehnung an efenn 'like, equal', oder an me. efenling, das Orrm *efennlinng geschrieben hätte. Dagegen glaube ich nicht, daIs $n n$ hier als etymologische entsprechung von $n l$ angesehen werden kann, wie Lambertz s. 24 vermutet.

Dafs Orrm mit seinen doppelkonsonanten die länge des konsonanten bezeichnen wollte, dafür spricht auch die art und weise, in welcher er sich der akzentzeichen bediente. Er hat wahrscheinlich nicht einmal gemerkt, dal's seine doppelschreibungen in sehr vielen fällen nicht nur die konsonantenquantitäten bezeichneten, sondern auch - dank den lautverhältnissen seiner sprache - die vokalquantitäten. Sonst hätte er wohl nicht lange vokale in geschlossener silbe mit akzenten bezeichnet (z. b. fór): der einfach geschriebene konsonant, der auf den vokal folgte, genügte ja, um die länge des vorangehenden vokals anzuzeigen. Dals er zur bezeichnung der vokalquantitäten akzente (und häkchen) überhaupt verwendete, deutet wohl auf dasselbe hin. Denn es wäre doch unmethodisch von ihm, wenn er durch so verschiedene mittel, wie akzente (bezw. häkchen) und doppelschreibungen der konsonanten, ganz denselben zweck verfolgen wollte, so lange eines von diesen mitteln (akzente und häkchen) diesem zweck völlig entsprochen hätte. Und unmethodisch war Orrm doch nicht!

Betreffs der Orrmschen akzente und häkchen ist neuerdings eine von der landläufigen meinung abweichende ansicht von Deutschbein (Archiv CXXVI s. 49 ff., CXXVII s. 308 ff.) ausgesprochen worden. Nach ihm bezeichnen die akzente reduzierte länge, bezw. halblänge, die häkchen überkürze.

1) Die schreibungen pane, whane (statt panne, whanne (Effer, Anglia Anz. VII s..179) beruhen wohl auch auf konsonantenkürzung in unbetonter stellung. Über die angeblichen schreibungen šne, kð̌ne, sŭne, wð̌nenn siehe Effer a. a. 0. und s. 167 anm. 
Ich kann mich der auffassung Deutschbeins nicht anschliefsen. Was zuerst die akzente betrifft, wäre es doch a priori zu verwundern, wenn er durch sie eine reduzierte quantität bezeichnen wollte - dazu hätte doch Deutschbein etwaige gegenstücke aus irgend einer sprache aufweisen sollen!

Dagegen halte ich, was die akzente betrifft, die grundlagen der Deutschbeinschen darstellung in einer hinsicht für im grofsen und ganzen richtig: ich glaube nämlich, dafs die langen vokale oder gewisse lange vokale zu Orrms zeit unter gewissen umständen reduziert waren oder mit reduzierter länge bezw. halblänge (vielleicht sogar mit kürze) ausgesprochen werden konnten. Ich glaube aber nicht, dals Orrm mit seinen akzenten diese reduzierte länge bezw. halblänge bezeichnen wollte: dagegen finde ich die annahme psychologisch gut begründet, dafs Orrm unter umständen mit seinen akzenten dem leser die nach seiner ansicht richtige aussprache mit langem vokal energisch einprägen wollte. Besonders einleuchtend finde ich diese erklärung der äufserst häufigen zwei-, ja dreifachen (wie es scheint niemals einfachen!) 1) akzente vor auslautendem $t$ (z. b. $u^{\prime \prime} t, u^{\prime \prime \prime} t, z^{\prime \prime} t, z^{\prime \prime \prime} t, h_{a}^{\prime \prime} t, h_{a}^{\prime \prime} t t$, butt, gat, ga $t$, wät, loet $t$, fot, silt, si't, swet, wallhat): wie die sprachgeschichte uns lehrt, läfst sich eine neigung zur reduktion der quantität des vokals hier schon früh spüren, und es ist wohl anzunehmen, dals zu Orrms zeit neben der sorgfältigeren aussprache mit länge auch halblänge (oder vielleicht sogar kürze) vorkam.

Es ist nicht unwichtig, dafs zwei oder drei akzente sich (nach Deutschbein) in der regel nur bei auslautendem $t$ finden, ${ }^{2}$ ) und dals hier einfacher akzent niemals vorzukommen scheint. Denn gerade in dieser stellung lälst sich eine verhältnismälsig frühe reduktion der vokalquantität beweisen. Ich finde deshalb Trautmanns erklärung der akzente in dieser stellung (Anglia XVIII s. $377 \mathrm{f}$.) ganz zutreffend. ${ }^{3}$ ) Es ist also

1) Vgl. Deutschbein, Archiv CXXVI s. 56.

2) Daneben ganz selten vor $d$ : bäd (1 mal), göd (1 mal); einmal vor intervokalischem $d$ : röde.

3) "Dafs Orrm einen, zwei oder sogar drei längestriche über diese vokale setzt, erklärt sich sehr einfach, wenn man annimmt, dals die neigung zu kürzen schon am anfange des 13. jahrh. eingesetzt hatte, und dafs Orrm 
meiner ansicht nach ganz klar, dals Orrm mit seinen zweifachen und dreifachen akzenten die länge des vokals besonders energisch hervorheben wollte. Es fragt sich nun, was er mit den einfachen akzenten bezweckte. ${ }^{1}$ )

Es versteht sich ohne weiteres, dafs in solchen fällen, wo er einfachen akzent schrieb, andere verhältnisse vorgelegen haben müssen als in denen, wo er zwei oder drei akzente verwendete; sonst hätten wir eine so klare scheidung zwischen den beiden kategorien nicht zu erwarten.

Es mufs nun zunächst hervorgehoben werden, dals die einfachen akzente verhältnismälsig selten sind und aufserdem nicht mit derselben konsequenz auftreten wie die zwei- oder dreifachen, z. b. in $u^{\prime \prime}\left(\right.$ ("ut $^{\prime}$ ). Das material in geschlossener silbe ist sogar recht dürftig: bód (1 mal), fór $(17 \mathrm{mal})$, tór (2 mal), anán (3 mal), mán (1 mal), fír (3 mal), rás (1 mal) náfrór (1 mal), pópre (3 mal), tálde (1 mal), tácness (1 mal; zweifelhaft). In offener silbe tritt der akzent etwas häufiger auf, wie die sammlungen bei Deutschbein (Archiv CXXVII s. 308 f.) zeigen. ${ }^{2}$ ) In fremdnamen (hebräischen oder griechischen ursprungs) ist der akzent sowohl in geschlossener als in offener silbe häufiger.

mit seinen strichen sagen will: Das wort fot, gat, ut, zet usf. hat langen vokal, zwar manche leute, die nicht ordentlich Englisch können, sagen f $\gamma t$, aber du freundlicher leser wirst $f \bar{\partial} t$ sprechen." Dazu will ich nur hinzufügen, dafs Trautmann nicht wufste, dals in dieser stellung einfacher akzent nicht vorkam. Es ist auch nicht so sicher, dafs fot zu dieser zeit geradezu mit kürze ausgesprochen werden konnte.

1) Ich habe oben fälle wie püt $(<p u(h) i t)$, het $(<$ he $(h) i t)$, zhöt $(<$ zho $(h) i t)$ absichtlich nicht besprochen, da die doppelten Akzente hier vielleicht anders erklärt werden können. So fassen Jessen a. a. 0. s. 207, Kluge, Grdr. $I^{2}$ s. 1058 den zweiten akzent als elisionszeichen auf.

2) Die betreffenden wörter sind: háte (adj.), hátenn (part.), láte (sb.), látepp (vb.), loete (vb.), loetenn ( $\mathrm{vb}$.$) , sóte (sb), léte (prät.), létenn (prät.),$ forrlétenn, swétepb (vb.), efennméte, write, wrí(t)tenn, lítell, wite (sb.), móte, róte (sb.), úte, lútenn (inf.), rédenn (pl. präs.), bédebp, wíde, síde, góde (adj.), áne, áness, náness, téne (sb.), téne (zahlw.), séne, béne, míne, tíne (= bine), pine, sóne, túne, áre, árenn, láre, máre, fére (sb. 'power'), hére (adv.), tíme, ríme, cóme, cómenn, lóme, wáke, tákenn, forrtákenn, tókenn, mále, whíle, rapenn, nápe, úpe, síze, drízcrafftess, lifess, wózhe. Hierher gehört hólepb 9319 (sieh NED.; anders Deutschbein). Es möge hier bemerkt sein, daIs diese wörter im allgemeinen nur 1-3 mal mit akzent vorkommen. Einzige ausnahmen sind létenn $(5 \mathrm{mal})$, áne $(6 \mathrm{mal})$, hére $(5 \mathrm{mal})$, tíme $(20 \mathrm{mal})$. 
Nach Deutschbein bedeutet der einfache akzent in geschlossener silbe wie in fór 'fuhr' reduzierte länge (d. h. eine quantität zwischen länge und halblänge); denselben wert will nun Deutschbein auch den akzenten in offener silbe beimessen. Diese kürzung soll - wenn sie nicht auf unbetontheit oder minderbetontheit beruht - in geschlossener silbe lautgesetzlich und dann auf analogischem wege auf die vokale in offener silbe übertragen worden sein. ${ }^{1)}$

Gegen Deutschbeins annahme spricht gewissermaisen der umstand, dafs die fälle in offener silbe zahlreicher sind als in geschlossener. Wenn wir von dem 17 mal belegten fór absehen, ist der rest ja recht kümmerlich. Und dieser rest reicht nicht aus, um die vielen fälle in offener silbe, als auf analogie nach den anderen beruhend, $\left.{ }^{2}\right)$ zu erklären. Es scheint da methodischer, oder wenigstens ebenso methodisch, von den fällen in offener silbe auszugehen, und wenn wir das tun, taugt Deutschbeins theorie nichts mehr. Wir müssen dann annehmen, dafs Orrm mit seinen einfachen akzenten - gerade wie mit den zwei- bezw. dreifachen - länge bezeichnen wollte; sein einziger grund, sich mit einem einfachen akzent zu begnügen, war, dafs hier keine von ihm verworfenen aussprachevarianten mit kürzung existierten.

Es ist wahr, dafs in sehr vielen fällen auf den mit akzent versehenen vokal ein dental folgt (dieser umstand ist für Deutschbein vom grö(sten gewicht). Das kann aber zufall sein; man darf auch nicht vergessen, dafs gerade das wort, in welchem der akzent am häufigsten auftritt (tíme $20 \mathrm{mal}$ ), nicht einen dental nach dem stammvokal enthält, und es geht doch nicht an, dieses wort $\mathrm{zu}$ den satzminderbetonten $\mathrm{zu}$ rechnen, wie es Deutschbein tut, um eine erklärung des akzents zu finden.

Weshalb nun gerade time so oft mit akzent geschrieben ist, läIst sich nicht leicht ermitteln. Was dagegen das häufige

1) Für einige fälle nimmt Deutschbein auch lautgesetzliche kürzung in offener silbe an (Archiv CXXVII s. 311).

2) Vokal $+t$, z. b. é in létenn, soll durch den einflufs einsilbiger formen (z. b. des sing. l"ct mit halblänge) reduzierte länge erhalten haben. Wer an Deutschbeins erklärung von lët nicht glaubt, kann aber seine erklärung von létenn nicht annehmen. 
fór betrifft, liegt die vermutung nahe, dals der akzent hier zur unterscheidung von der präposition forr diente. Der einwand Deutschbeins, Archiv CXXVI s. 55, dals in forr die kürze durch $r r$ bezeichnet war, 1) fällt nicht sehr ins gewicht, wenn man beweisen kann, dafs die doppelkonsonanten bei Orrm lediglich konsonantenlänge bezeichneten und dals Orrm selber sich die tatsache kaum klar gemacht hatte, dafs sie unter gewissen umständen auch über die quantität des vorangehenden vokals aufschlufs geben konnten.

DaIs in den hebräisch-griechischen namen der akzent so häufig vorkommt, ist leicht zu erklären. Orrm hatte ja gewifs gründe anzunehmen, dafs die mehrzahl seiner leser mit der aussprache dieser namen wenig vertraut waren. Er wollte ihnen nun durch seine akzente die seiner ansicht nach richtige

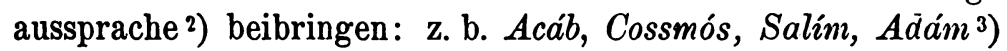
(neben Adam), Sálèmannes, Sabá. Zwei akzente setzte Orrm bei Goliät, Jaf'eth, $\left.{ }^{4}\right)$ wahrscheinlich weil er von diesen namen oft eine unrichtige aussprache gehört hatte.

Was Orrm mit seinen häkchen bezeichnen wollte, ist für unsere hier erörterten fragen ziemlich gleichgültig. Ich will deshalb diese frage nur kurz streifen. Deutschbein glaubt, dafs Orrm mit ihnen überkürze des betreffenden vokals andeutete. Das kommt mir nun nichts weniger als wahrscheinlich vor. Nehmen wir an, dafs Orrm mit seinen akzenten länge bezeichnen wollte, so bleibt uns eigentlich nichts anderes übrig, als seine häkchen einfach als kürzezeichen aufzufassen. Und ich glaube, das geht ganz gut an. Es ist wahr, daIs die häkchen wie die akzente nicht überall stehen, wo man sie zu erwarten hätte. Auch das kann zufall sein. In vielen fällen wurde das häkchen gesetzt, um das wort von einem anderen mit langem vokal zu unterscheiden z. b. tăkenn (vgl.

1) Deutschbeins worte a. a. 0.: "Zur unterscheidung von der präposition forr kann der akzent auf fór auch nicht dienen, da for bei Orrm natürlich als forr erscheint" sind wohl so zu deuten.

2) Wie Orrm zu dieser aussprache gekommen ist, geht uns hier wenig an. Sie beruhte wohl im allgemeinen auf französischem einflufs.

8) Diese beispiele sind an und für sich genug um zu beweisen, dafs Orrm den einfachen endkonsonanten nicht als ein zeichen der länge des vorangehenden vokales auffaiste.

•) Vgl. Reichmann, Die Eigennamen im Orrmulum, s. 84. 
tákenn), vitenn (*witenn ist zufällig nicht belegt, nur das part. prät. witenn), und von dem infinitiv wurde das häkchen auch auf formen wie takepp übertragen.

Man muls auch mit der möglichkeit rechnen, dafs die häkchen und akzente erst nachträglich eingesetzt wurden, und man kann Orrm nicht verdenken, wenn er von ihnen keinen konsequenten gebrauch machte. ${ }^{1}$ )

Zuletzt einige einzelbemerkungen.

1. tōr 'hard, difficult'. Die zusammenstellung mit altn. tor- 'an inseparable prefix in Icel. denoting difficulty' weist Brate, PBB. X s. 59, wegen der vokallänge zurück. Ich glaube jedoch, dâs es möglichkeiten gibt, das me. adjektiv aus dem nordischen praefix herzuleiten.

Im Altnordischen hat das praefix zwei formen tor- und tyr-, altschwed. tor- und tor. Diese doppelformen beruhen darauf, dals das praefix unter gewissen wohlbekannten umständen bald starktonig, bald tieftonig war. Das ursprünglich unbetonte tor- scheint früh auch in starktoniger stellung gewöhnlich geworden $\mathrm{zu}$ sein. Die entgegengesetzte bedeutung hatte das altn. auð- 'leicht' (= ae. éað-, $\bar{e} \delta$-). Beispiele sind: altn. torbreytiligr, torbreytr 'schwierig $\mathrm{zu}$ überwinden', torbenn 'schwierig mit bitten $\mathrm{zu}$ bewegen', torbstr 'schwierig zu sühnen', torfenginn, torfengr 'schwierig zu gewinnen oder erlangen', torfyndr 'schwierig zu finden', torfóra, torfóri, torleiði 'platz wo es schwierig ist zu passieren', torfórr 'schwierig zu passieren', torkendr 'schwierig wiederzuerkennen', torkenna 'unkenntlich machen', torsveig(J)r 'schwierig zu biegen', tortaliðr 'schwierig zu zählen', tortima 'zerstören', torvelda 'schwierig machen'. torvirðr 'schwierig zu schätzen oder würdigen' usw., tyrtryggia 'milstrauen', altschwed. torkanna, torkanna 'unkenntlich machen'. Über die nordischen formen, ihre erklärung und geschichte, siehe Noreen, Altisl. Gr. § 51, 1 a ; 68, 5; 69; 121 anm. 1; 139, 3, Altschwed. Gr. § 64, $3 ; 84$ anm. $7 ; 268$ anm. 2.

Nun scheint auch das Altenglische das praefix tor-gekannt

1) Man vergleiche die nachträgliche und bei weitem nicht konsequente behandlung, die eo im Orrmulum erfahren hat. Siehe Hale, Mod. Langu. Notes VIlI (1893) sp. 42 ff., Bülbring, Bonner Studien XVII (1905) s. 52 ff. 
$\mathrm{zu}$ haben (tor-bezete 'hard to get', tor-cierre 'hard to turn, hard to convert'); im Mittelenglischen habe ich nur das sicher aus dem Nordischen stammende torfére 'hardship, difficulty' gefunden. Es ist zwar möglich, dals tōr ein einheimisches englisches wort ist; für nordischen ursprung spricht aber ein anderer umstand, den ich gleich erwähnen werde.

Nun wäre es schon an und für sich möglich, aus tor-, dessen bedeutung ja lebendig war, ein adjektiv zu machen; vgl. Lehmann, Das Præfix uz-, besonders im Altenglischen (Kiel 1906) s. 83. Ich glaube aber, ein besonderer umstand hat diesen übergang erleichtert. Der gegensatz zu tor-war

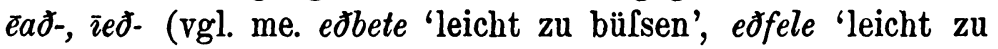
empfinden', eđzete 'leicht zu erlangen', eðlete 'gering zu achten, wertlos', eðsene 'leicht zu sehen'). Das entsprechende adjektiv war ae. nun nicht die $\mathrm{zu}$ erwartende form ${ }^{*} \overline{e p e}$ (adj.), wogegen das adverb regelmälsig $\bar{x} p e$ heilst. Das adjektiv lautet bei Orrm $\bar{a} p$ (z. b. 13026: $\bar{x} \not \bar{p}$ to shōewenn). Dieses könnte ja zwar aus dem adverb abstrahiert sein, in dem $-e$ als adverbialendung aufgefafst wurde, $\overline{\bar{a}} \delta$ also eine rückbildung ("backformation") war; aber es ist auch möglich, dals das præfix * $\bar{x} b$ - der form des adjektivs $\mathrm{zu}$ grunde liegt.

Aus diesen verhältnissen ist nun die folgende gleichung entstanden: $\bar{a} \delta$ - (præfix): $\bar{a} \delta$ (adj.) $=$ tor- (præfix): $\mathrm{X} ; \mathrm{X}=$ tor (adj.). Schwierig zu erklären ist nur die länge des vokals im adjektiv tor. Wie im Nordischen aus $u z$ - (neben or-, ur-, ur-, or-) ór entstanden ist (Noreen, Altisl. Gr. §69), so wäre auch ein nordisches *tór denkbar. Daneben möchte ich noch die folgende möglichkeit hervorheben: Der vokal im præfix wurde vor einem mit einem konsonanten anlautenden zweiten gliede lautgesetzlich gekürzt (vgl. aedmod neben addmod bei Orrm). Daraus konnte im Spätaltenglischen oder Frühmittel-

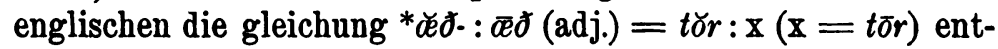

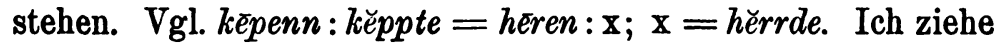
aber vor, $\bar{o}$ ungefähr in derselben weise wie $\bar{\imath}$ in schwed. $h \bar{\imath} n$ (oben s. $361 \mathrm{anm}$.) zu erklären: tōr war öfter unbetont und stand in der regel vor einem konsonanten. In kompositionsgliedern (bezw. vorsilben) vor einem konsonantisch anlautenden zweiten gliede und in unbetonten wörtern (bezw. vorsilben) trat in zahlreichen fällen lautgesetzlich vokalkürzung ein; daneben 
lebte auch die ursprüngliche aussprache mit langem vokal, be- sonders im simplex, wenn ein solches vorhanden war (vgl. z. b. me. chappmann neben chęp). Es lag also die versuchung nahe, aus dem præfix tŏr- ein adjektiv tōr zu machen.

Was nun für meine erklärung von tōr besonders spricht, ist die me. nebenform tere, das nach dem NED. mehrere male nach 1400 belegt ist, $\mathrm{z}$. b. tere to tellen 'difficult to tell'. Ich erkläre dieses direkt aus dem Ostnord. tor- 'difficult'. Bei Orrm hätte dies adjektiv wahrscheinlich *tēr gelautet. Da me. tere, wenn meine erklärung richtig ist, nordisch sein mufs ( $\theta$ ist durch den nordischen $R$-umlaut entstanden), scheint es ratsam, auch tōr aus dem Nordischen zu erklären. ${ }^{1)}$

2. topp wird von Wh.-H. mit 'rapacity, rapacious appetite' übersetzt und mit ae. $t \bar{o} \bar{b}$ 'zahn' zusammengestellt. Diese zusammenstellung ist, wie Brate PBB. X s. 59 hervorhebt, ${ }^{2}$ ) der quantitätsverhältnisse wegen unmöglich. Auch die von Jessen vorgeschlagene herleitung (Tidskr. for phil. og pæd I. s. 226) aus isl. tøð pl. von tað 'manure, dung' ist unmöglich.

Es ist nicht ganz sicher, dals das wort von Wh.-H. richtig übersetzt ist. Es begegnet an den folgenden stellen:

v. $7184 \mathrm{ff} .:$ Annd tohh swa pehh iss ned tatt he

Dredinng annd azhe sette

On alle pa patt lufenn topp

Annd woh annd unnsahhtnesse,

To don hemm follzhenn lazhe annd gripp

All pezzre cebore unnpannkess,

ziff patt tezz pezzre pannpess gripb

Ne kepenn nohht to follzhenn.

v. $9316 \mathrm{ff}$ : Jiff patt ze wel zuw lokenn

Fra clake annd sake, annd fra batt topb

$p_{\text {at follzhepp zifernesse. }}$

v. $10200 \mathrm{ff.:}$ Jiff patt ze wel zuw lokenn

Fra clake annd sake, annd fra patt topp

$\bar{p}_{\text {att follzhepp grediznesse. }}$

1) Wie Lehmann a. a. 0. hervorhebt, ist die möglichkeit nicht ganz ausgeschlossen, daIs auch ae. tor- aus dem Nordischen stammt.

2) Vgl. Lambertz s. 47. Anders Morsbach, Me. Gr. s. 67.

Anglia. N.F. XXV. 
Die zusammenstellung mit wōh 'evil, wrong, wickedness', unnsahhtnesse 'discord', clake annd sake 'injury and accusa- . tion' (sieh NED.) $\left.{ }^{1}\right)$ spricht nicht gerade für Wh.-Holts übersetzung von $t o b p$; es ist ferner wahrscheinlich, dafs ziferrnesse und grediznesse mit 'geiz, habsucht', höchstens mit 'begierde, lüsternheit' zu übersetzen ist. Das spricht auch gegen die bedeutung 'rapacious appetite'; dagegen wäre die bedeutung 'rapacity' schon möglich.

Obgleich also die bedeutung von to $p p$ sich nicht sicher feststellen lälst, möchte ich jedoch eine anknüpfung vorschlagen.

Ich halte topp für ein nordisches lehnwort. Altisl.-altnorw. *toð kann ich zwar nicht finden, aber im Ostnordischen ist ein solches wort sicher belegt: altdän. tåd 'hindernis, verhinderung', dän. dial. taad 'hindernis, verzug, verzögerung', taae 'bürde' (Molbech, Dansk Dialektlexikon, Dansk Glossarium), dazu das verb altdän. tothce (Jyske Lov.), tåde 'hindern, aufhalten' (Kalkar), dän. dial. taade 'aufhalten, hindern', taaєn adj. 'dünn, mager, milsraten, verkümmert'. Die schreibung tothor $\left.{ }^{2}\right)$ im jütischen gesetz beweist, dafs das nda. $\&$ (aa) aus

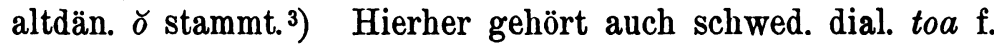
'last, beschwerde, kosten, übelstand, schwierigkeit' (Rietz). Das neutr. töje bei Rietz gehört wohl auch hierher.

Das ist meines wissens die einzige mögliche anknüpfung, um Orrms tobp zu erklären. Eine schwierigkeit bleibt noch $\mathrm{zu}$ beseitigen, die der bedeutungsfrage. Was ist das für ein to $p p$, das die habsucht oder begierde mit sich bringt? Un-

1) Ich bin nicht ganz davon überzengt, dafs clake 'injury, scathe' bedeutet. Die von Wh.-H. angegebene bedeutung 'accusation, fault, vice' und die von Dodd, A Glossary of Wulfstan's Homilies (1908) angenommene 'contention, strife' befriedigen auch nicht ganz. Die stelle bei Wulfstan (sacu and clacu, hol and hete) legt die vermutung nahe, dals es 'chatter, vain speech, slander' (wie hol) bedeutet und also mit claik (im NED.) zusammengehört. Andere erklärung bei Brate, PBB. X s. 37. Wie Brate hervorhebt, ist Orrm $9316 \mathrm{ff}$. eine wiedergabe von Lukas III, 14: Et ait illis: neminem concutiatis neque calumniam faciatis et contenti estote stipendiis vestris.

2) tothar ham swa at han ma aei burt (kumma) meth sine kostce Jy. Lov. II 76.

3) Die erklärung bei Lund, Ordb. ist, wie Kalkar hervorhebt, unmöglich. Dais die grundform * ${ }^{*} \varnothing \delta$ ist, bestätigt mir auch mein kollege 0 . . Friesen, mit dem ich die frage besprochen habe. 
willkürlich denkt man dabei an die bekannte stelle im ersten brief des apostels Paulus an Timotheus (VI, 9-10), die im Vulgata-text folgendermalsen lautet: Nam qui volunt divites fieri incidunt in tentationem et in laqueum diaboli et desideria multa inutilia et nociva, que mergunt homines in interitum et perditionem. Radix enim omnium malorum est cupiditas, quam quidam appetentes, erraverunt a fide et inserverunt se doloribus multis. Vielleicht bedeutet $t o p b$ eben diese schlinge der habsucht oder begierde. Vielleicht lieise sich patt topp patt follzhepp grediznesse (ziferrnesse) als die 'unzufriedenheit des geizigen' (vgl. Luk. III, 14: contenti estote usw.) auffassen. Es wäre vielleicht auch möglich, to $\not p$ einfach mit 'hindernis, verzug, zauderhaftigkeit', daher 'unbulsfertigkeit' zu übersetzen.

3. comm præt. zu cumenn soll, wie die præt. drohh, hoff, nach Lambertz s. 46 eine kürzung nach analogie der starken verba der IV. und V. klasse erfahren haben. Brate PBB. X 8. 38 sagt zu comm: "Vielleicht ist eine teilweise angleichung an den plural cōmenn anzunehmen, so dals die quantität des vokals im sg. erhalten, die qualität desselben aber dem plural gleich gemacht ist." Es scheint mir wahrscheinlicher, dafs die kürzung auf satztiefton beruht (s. b. drazhenn fórp 11907, 11929, drazhenn úpp 16856, hefenn úpp 14676, cumenn dún 12095). Was besonders comm betrifft (es heifst bei Orrm stets so, dagegen droh $15 \mathrm{mal}$, drohh $4 \mathrm{mal}$, hof $3 \mathrm{mal}$, hoff 1 mal), lälst es sich aber auch auf nordischen einfluls zurückführen. Was Brate gegen eine solche annahme angeführt hat (a. a. 0.), überzeugt mich nicht. Sowohl im Altwestnordischen als im Altschwedischen war das prät. $k \check{c} m$ sehr häufig, im Altschwedischen war es sogar die normalform. Vgl. Noreen, Altisl. Gr. § 486, Altschwed. Gr. § 356. ') Man vergleiche das nord. Lelunwort cōme 'coming, advent' bei Orrm, dals ich in meinen Scand. Loan-words s. 11 anm. 2 richtig erklärt zu haben glaube. ${ }^{2}$ ).

4. Drihhtin wird von Orrm vollkommen als ein eigenname betrachtet. Es hat niemals den bestimmten artikel (also z. b.

1) Zur erklärung der altnord. præteritalform $k ð m$ siehe Ljungstedt, Anmärkningar till det starka preteritum i germanska språk, Uppsala 1887, s. $112 \mathrm{f}$.

2) Dafs ich an Deutschbeins erklärung der schreibungen cóme, cómenn nicht glaube, brauche ich wohl nicht zu sagen. 
Drihhtin Godd 16720, aber pe Laferrd Godd 16712); auch die beispiele aus anderen denkmälern im NED. haben keinen bestimmten artikel. Das gilt auch für alts. drohtin, ahd. truhtĩn (trohtîn); letzteres wird sogar oft wie ein eigenname flektiert (Braune, Ahd. Gr. $§ 195$ anm. 1), indem es den akk. sg. truhtînan (neben truhtîn) hat. Das wort bietet in bezug auf seine form auch im Althochdeutschen schwierigkeiten, die wohl damit zusammenhängen, dals es als literarisches wanderwort seine eigene geschichte haben dürfte. 1) - Es scheint mir unter diesen umständen nicht möglich, -inn in Orrms Drihhtin auf den einflufs von me. almihtīn zurückzuführen, wie es Kluge tut (Pauls Grundr. I ${ }^{2}$ s. 1059), ${ }^{2}$ ) da diese erklärung sich nicht auf ahd. truhtîn anwenden lälst. Die endung aus ae. formen wie dryhten mīn, frēa mīn zu folgern, spricht mich auch wenig an. Ich ziehe deshalb vor, in -in (statt -en) das lateinisch-romanische, gerade in personennamen so häufige suffix (z. b. Awwstin, lat. Augustinus) zu erblicken. ${ }^{3}$ ) Ich vermute, dals gerade ein solches wort der gegenstand volksetymologischer umwandlungen, die auf spintisierungen mönchischer gelehrsamkeit gefuIst waren, werden konnte. Die ziemlich internationale endung -inn liegt wohl auch in den kontinentalen formen vor.

Als junger student nahm ich einmal deutsche konversationsstunden bei einer deutschen dame. Ich erinnere mich noch lebhaft daran, wie ich mich darüber ärgerte, dafs sie mir gar zu wenig kenntnisse und fassungsgabe zutraute. Sie redete mich sogar ungefähr in dieser weise an: $g \hat{u}-t e ̂ n n n$ mörr-gěnn, wí gét ếss î-nénn. Sie sprach also - um mir keine silbe verloren gehen zu lassen - genau so wie der gute Orrm

1) Vgl. Franck, Altfränkische Grammatik s. 107.

2) "Das me. adjektiv almihtín (genet. godalmihtines Vices a. V. 41) mit schwerer endung, verdankt seinen ursprung dem ae. akkusativ almihtizne: das seit Chro. 1137 für ae.dryhten, drihten auftretende, in Hal.-Maid., Jul., und Marh. herrschende drihtín, drightín entsteht unter dem einflufs von almihtín oder von ae. formeln wie drihten mîn, fréa min (as. drohtîn, ahd. trohtin scheinen auch sekundäres $\hat{\imath} \mathrm{zu}$ haben)."

8) Vgl. Björkman, Archiv CXIX \&. 36. - Über Orrmīn siehe Björkman, Archiv CXXIII 8. 23 ff. 
schrieb. Und wie sie sprach, so liest noch mancher lehrer und manches schulkind. Ich glaube, ein besuch in der kleinkinderschule - sei es in Deutschland, England oder Schweden - würde leicht den leser von der richtigkeit meiner theorie überzeugen. Wer einmal die konsonanten, die in der ungezwungenen rede kurz sind, bei den leseübungen in der kleinkinderschule hat lang aussprechen hören, der glaubt nicht mehr, dafs die doppelschreibung der konsonanten bei Orrm lediglich vokalkürze bezeichnete.

Wenn noch in unseren tagen lehrer und schulkinder so sprechen und lesen können, wie Orrm schrieb, da fragt man sich: weshalb hat nicht Orrm selber so sprechen können, wie er schrieb? Ich meine nicht im vers, geschweige denn in der natürlichen ungezwungenen rede, sondern nur gelegentlich, als er seine eigene aussprache genau analysieren und seinem eigenen sprachgefühl klar machen wollte, indem er jede silbe betonte, wie es manche leute bei ähnlichen gelegenheiten noch heute tun. Noch heute glauben gebildete, aber phonetisch ungeschulte leute, dafs sie in der natürlichen rede jedes unbetonte wort, jede unbetonte silbe so aussprechen, wie sie diese wörter und diese silben in isolierter stellung, d. h. unter dem hochton, aussprechen. So auch Orrm. Sein orthographisches prinzip war rein phonetisch: er wollte so schreiben wie er sprach. Es galt ihm aber ausfindig zu machen, wie er wirklich sprach. Das war nun keine leichte sache. Sogar geschulte phonetiker des 19 . oder 20. jahrhunderts haben sich über ihre eigene aussprache getäuscht. Orrm schrieb nicht so wie er sprach - wenigstens nicht so, wie er im vers oder in der natürlichen rede sprach - sondern er schrieb so wie er zu sprechen glaubte. Das tun noch unsere modernen phonetiker. Und mehr können wir weder von dem einen noch von dem anderen verlangen.

UPPSALA.

ERIK BJörkman. 